\title{
Topology Controlled Channel Assignment Scheme with Power Control in MRMC Wireless Mesh Networks
}

\author{
Sang Hyun Lee and Hong Shik Park
}

\begin{abstract}
Wireless mesh networks are being deployed not only in various industrial fields but also in particular service required areas such as wireless access networks, disaster area, hot spot zone, and the area where fast network construction is required. In these cases, since the mesh node power supply is limited and the channel interference range is related to the transmission power of the nodes, the proper transmission power should be selected to maintain the network connectivity and minimize inter-channel interference. In the paper, the proposed heuristic algorithm, called Topology Controlled Channel Assignment with power control (TCCA), measures the received and transmitted power using a beacon packet and allocates the best channel with the highest SINR value for the HWMP (Hybrid Wireless Mesh Protocol) routing protocol in a real-time basis. We evaluate the networks throughput, end-to-end delay performance of the TCCA algorithm using the NS-3 network simulator. From the simulation studies, we show that the TCCA algorithm outperforms a SINR based channel assignment with the same transmission power.
\end{abstract}

Index Terms-Multi-radio multi-channel wireless mesh networks, channel assignment, power control, real-time based algorithm.

\section{INTRODUCTION}

Wireless Mesh Networks (WMNs) have emerged as a suitable networking technology for covering the vast area with excellent characteristics such as simple deployment, efficient maintenance, scalability, and connectivity [1], [2]. Because of these features, WMNs can be adapted in the areas where it is difficult to construct wired networks or where immediately network construction are required (such as wireless access networks, area where the network was destroyed by disaster, area where an additional network need to be built for unexpected heavy traffic, etc).

There are several issues to be considered when constructing the WMN in the above environment. First, it is important to reduce the power consumption of each node because WMN is difficult to provide stable power supply through the wired connection. By the Friis's formula, the received power is proportional to the transmit power of neighbor node and inversely proportional to the square of the distance, if the spacing between two nodes increases two

Manuscript received January 5, 2017; revised April 17, 2017. This work was supported by Institute for Information \& communications Technology Promotion (IITP) grant funded by the Korea government (MSIP) (B0101-16-1270, Research on Communication Technology using Bio-Inspired Algorithm)

Sang Hyun Lee and Hong Shik Park are with the School of Electrical Engineering, Korea Advanced Institute of Science and Technology (KAIST), Daejeon, South Korea (e-mail: capricorns@kaist.ac.kr, park1507@kaist.ac.kr). times, then the power of the transmitting node must be increased four times. Consuming a lot of power to maintain connectivity with the distant neighbor nodes can cause network performance degradation due to power outages in the limited power supply. Therefore, power consumption in WMN should be minimized by determining the proper transmission power for neighboring nodes according to the WMN topology.

Second, increasing the transmission power to maintain network connectivity causes more channel interference in some channels. Appropriate control of transmission power can improve network capacity by reducing channel interference, and enhance the spatial channel usage efficiently. In this paper, we propose an algorithm called Topology Controlled Channel Assignment with power control (TCCA) that maintains connectivity between neighboring nodes, reduces channel interference in some channels and minimizes the transmission power for multi-radio and multi-channel WMN. The real-time based TCCA algorithm measures the received power of each node using the beacon packets and determines the transmission power in the distributed manner Moreover, the TCCA algorithm calculates a SINR (signal to interference and noise ratio) value using measured information and assigns a channel with the lowest interference in the centralized manner.

The rest of the paper is organized as follows. An overview of the related work is discussed in Section II. In section III, we describe the problem formulation and system model. The proposed TCCA algorithm and its performance verification are presented in Section IV and Section V, respectively. Finally, we conclude the paper in Section VI.

\section{RELATED WORK}

There are two types of channel interference models which are a protocol model and a physical model [2]. The protocol model considers interference and transmission range. On the other hand, the physical model computes SINR from incoming received power. The SINR value must be larger than the threshold value for successful transmission and reception. As, the physical model is more realistic than the protocol model, we use the physical model. Most of the channel assignment algorithms in [2] are traffic aware channel assignment. However, the traffic aware channel assignment method cannot operate stably, and the channel allocation results can be changed according to the traffic type, and the channel switching time cannot be ignored. So, traffic independent channel assignment algorithms can operate more stably.

The authors in [3] suggest the centralized channel 
assignment algorithm termed CLICA which is greedy heuristic with the graph-theoretic formulation. The objective of [3] is to minimize maximum interference while controlling topology and preserving the network connectivity using the protocol interference model. However, this algorithm does not consider power consumption and does not use the physical model for a realistic environment. The authors in [4] propose Topology-controlled Interference aware Channel Assignment (TICA) which is a centralized and quasi-static channel assignment technique. Their approach is topology control based on power control to assign channels with low interference by guaranteeing network connectivity. However, their network topology does not generate multipath due to shortest path tree based channel assignment. It also does not use the physical model.

The WMN standardization document released in 2012 [5] specifies Hybrid Wireless Mesh Protocol (HWMP) as an MAC layer routing protocol. The HWMP path selection protocol has a proactive mode and a reactive mode. The proactive mode routing is a root-based routing protocol that establishes a routing path through the only root node. Whereas, the reactive mode routing is an on-demand based routing protocol that generates a route according to flow request. Our TCCA algorithm is designed to maximize the HWMP path selection performance by reinforcing the connectivity of the root node and preventing unnecessary links generation.

\section{PROBLEM FORMULATION}

In this section, we describe problem formulation and a system model. Also, we present its notations.

First, we formulate WMN as a directed graph $G=(V, E)$ by a given mesh nodes information, where $V\left(v_{i} \in V, i \in N, N=|V|\right)$ is a set of mesh nodes and $E\left(e\left(v_{i} \rightarrow v_{j}\right) \in E, v_{i} v_{j} \in N\right)$ is a set of links between two mesh nodes. Using a measured received power $\left(R P_{v_{i}}\right)$ table, a topology graph $G_{T}=\left(V, E_{t}\right)$ that minimizes transmit power and shows the state of connections with neighboring nodes is obtained through the graph $G$. Finally, a channel assignment graph $G_{C A}=\left(V, E_{c a}\right)$ is obtained by the proposed algorithm and the topology graph $G_{T}$.

We only consider the backhaul wireless mesh network. So, we assume that each mesh node communicates with its mobile client users over the additional interfaces using different bandwidth. For example, a mesh backhaul network consists of $5 \mathrm{GHz}$ bands and a connection between mesh node and client consists of $2.4 \mathrm{GHz}$ bands. One of mesh nodes which is located in the edge of the network topology acts as a gateway mesh node to connect the Internet and mesh backhaul network. Each mesh node is equipped with $|K|$ radio interfaces that operate half-duplex omnidirectional antennas, where $K\left(k_{i} \in K, i=2, \ldots,|K|\right)$ is a set of interfaces. All interfaces of the mesh node have the same transmission power. A $C\left(c_{i} \in C, i=2, \ldots,|C|\right)$ is an available non-overlapping orthogonal channel set. The number of channels $(|C|)$ must be greater than or equal to the number of interfaces $(|K|)$.

There are two type of interference models which are the physical model and the protocol model. The protocol model which is much simpler than the physical model represents interference by expressing whether there is a node within interference range or not. On the other hand, the physical model is more accurate by computing the channel SINR value of neighboring nodes. Because of that reason, we use the SINR based physical model to assign a channel. SINR of each channel of each node is calculated as follows.

$$
\operatorname{SINR}_{v_{j}}^{c}\left(v_{i}\right)=\frac{P^{c}\left(v_{j} \rightarrow v_{i}\right)}{P_{\text {noise }}+\sum_{k \in V, k \neq i, j} P^{c}\left(v_{k} \rightarrow v_{i}\right)}
$$

The above equation means the SINR of node $v_{i}$ received from node $v_{j}$ at channel $c$, where $P^{c}\left(v_{j} \rightarrow v_{i}\right)$ is the received power of node $v_{i}$ from node $v_{j}$ at given channel $c$ and $P_{\text {noise }}$ is noise figure.

In the TCCA algorithm, each node measures and stores the received power and transmission power from other mesh nodes using the beacon packet. The measured information is stored at the received power $\left(R P_{v_{i}}\right)$ table as shown in Fig. 1.

That table stores the received power of all mesh nodes according to the transmission power steps. If each received power is greater than an energy detection threshold $\left(P_{e d}\right)$, then that neighbor node can communication with the current node.

The TCCA Algorithm creates the topology adjacency matrix ( $T$ ) with topology graph $G_{T}$ based on $R P_{v_{i}}$ and the number of interfaces. This directional matrix $T$ is not symmetric since the transmission powers of both nodes are not the same. If received power of node $v_{i}$ from node $v_{j}$ is greater than the $P_{e d}$, then node $v_{i}$ can transmit a packet to node $v_{j}$ and the value of $T_{i j}$ equals 1 , otherwise, 0 . A channel assignment matrix ( $C A$ ) can be obtained at the channel assignment stage. The $C A$ matrix is symmetric and the value of $C A_{i j}$ represents channel $I D(c)$ between node $v_{i}$ and $v_{j} . P_{T}$ is transmission power of all nodes obtained by topology graph $G_{T}$. The TCCA is the traffic independent channel assignment algorithm.

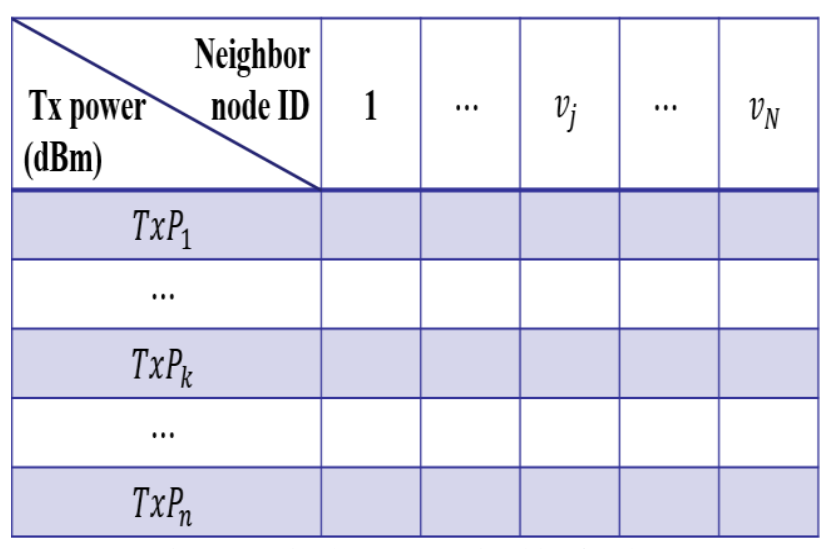

Fig. 1. Received power (RPvi) table of node $v_{i}$ 


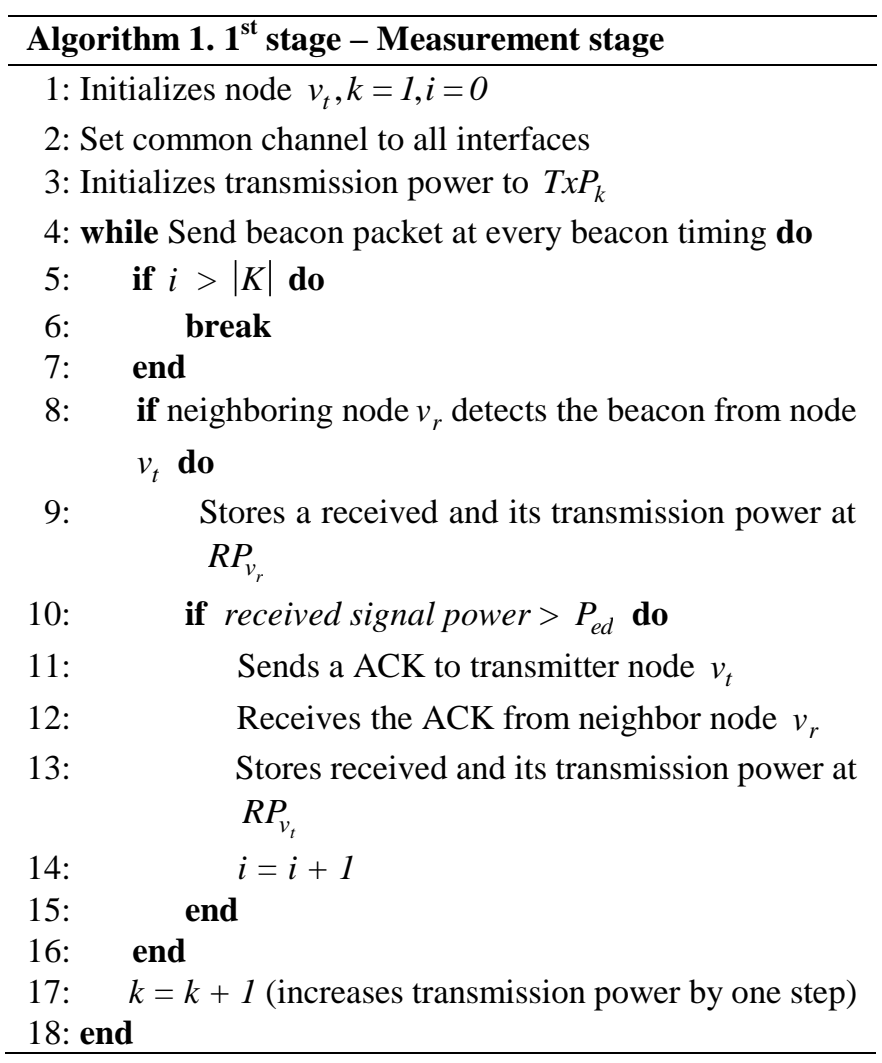

\section{PRoposed TCCA Algorithm}

In this section, we develop a heuristic algorithm called Topology Controlled Channel Assignment with power control (TCCA) algorithm for determining transmit power of a node according to topology and assigning the lowest interference channel. The TCCA algorithm is a real-time based channel assignment algorithm. This algorithm measures the received power of each node and determines the transmission power with the distributed concept. Also, it calculates a SINR value and assigns a channel in centralized concept.

The TCCA algorithm consists of by two stage. The first stage is the measurement stage that measures the received power of each node according to transmission power steps. The second stage is the channel assignment stage that determines transmission power and assigns a channel of each node based on the measured information of the first stage.

\section{A. 1st Stage - Measurement Stage}

The first stage is the measurement stage. The algorithm 1 is an algorithm for measuring and storing the received power and the transmission power for each node using the beacon packet that detects neighboring nodes. The algorithm 1 is executed at each node. The 8th to 11th commands line of the algorithm 1 are executed by the receiving nodes, and the other commands are executed by the transmitting node. For simplicity, we express $v_{t}$ and $v_{r}$ as transmitter and receiver node, respectively.

First, it sets the interfaces of all mesh nodes to the common channel $I D$ in order to measure transmitting and receiving power. Then, a beacon packet is transmitted at every beacon timing. When the neighboring node $v_{r}$ detects the beacon packet, it updates the $R P_{v_{r}}$. If received power is greater than $P_{e d}$, the node $v_{r}$ sends an ACK packet to the transmitter node $v_{t}$ and the node $v_{t}$ updates the $R P_{v_{t}}$. Until the number of received ACKs is greater than the $|K|$, transmitter node $v_{t}$ increases transmission power by one step and transmits a beacon packet again.

\section{B. 2nd Stage - Topology Controlled Channel Assignment Stage}

The second stage is the topology controlled channel assignment stage. The algorithm 2 creates the topology graph $G_{T}$ based on the measured transmission and received power, and performs channel assignment based on the generated $G_{T}$. The algorithm 2 is executed in the GW (gateway) mesh node.

First in the algorithm 2, $C A$ and $T$ is initialized by 0 , then the GW mesh node collects the measured received and transmitted power $\left(R P_{v}\right)$ table of all nodes. The $3^{\text {rd }}$ to $11^{\text {th }}$ command lines generate the topology $G_{T}$ that minimizes transmission power consumption based on measured $R P_{v}$ of each node.

In the case of GW node, since traffic is concentrated due to connections with the external Internet, it should be connected with as many neighbor nodes as possible. Therefore, the connection with the neighbor node of the GW node is reconfigured at the 12th command line. Also, for the above reason, the GW node performs channel assignment first as shown in the 13th command line. The proposed TCCA algorithm divides the channel assignment order into non-edge nodes and edge nodes. The non-edge nodes which are located in the central position of the WMN are first allocated a link with the best possible channel because the traffic is concentrated more compared to the located at the edge nodes of the WMN, as shown in the 14th to 19 th command lines.

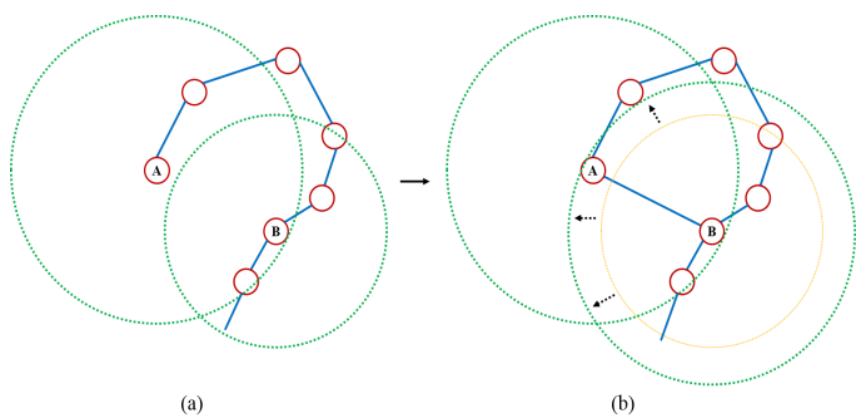

Fig. 2. Description for transmission power extend node B.

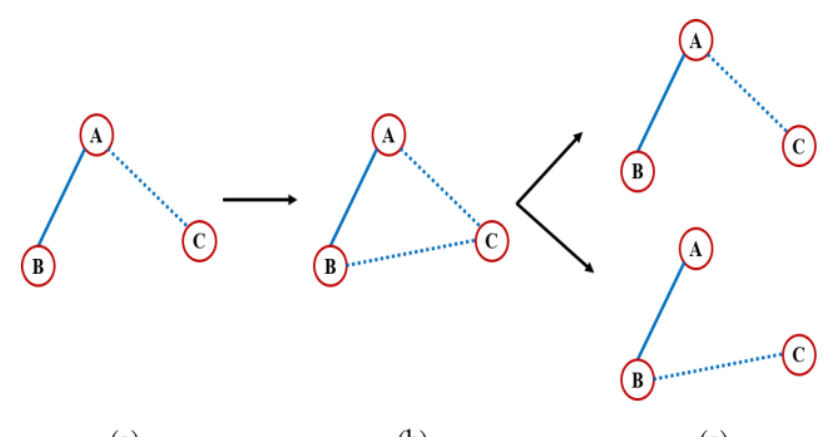

(a)

(b)

(c)

Fig. 3. Description for preventing triangle topology creation. 


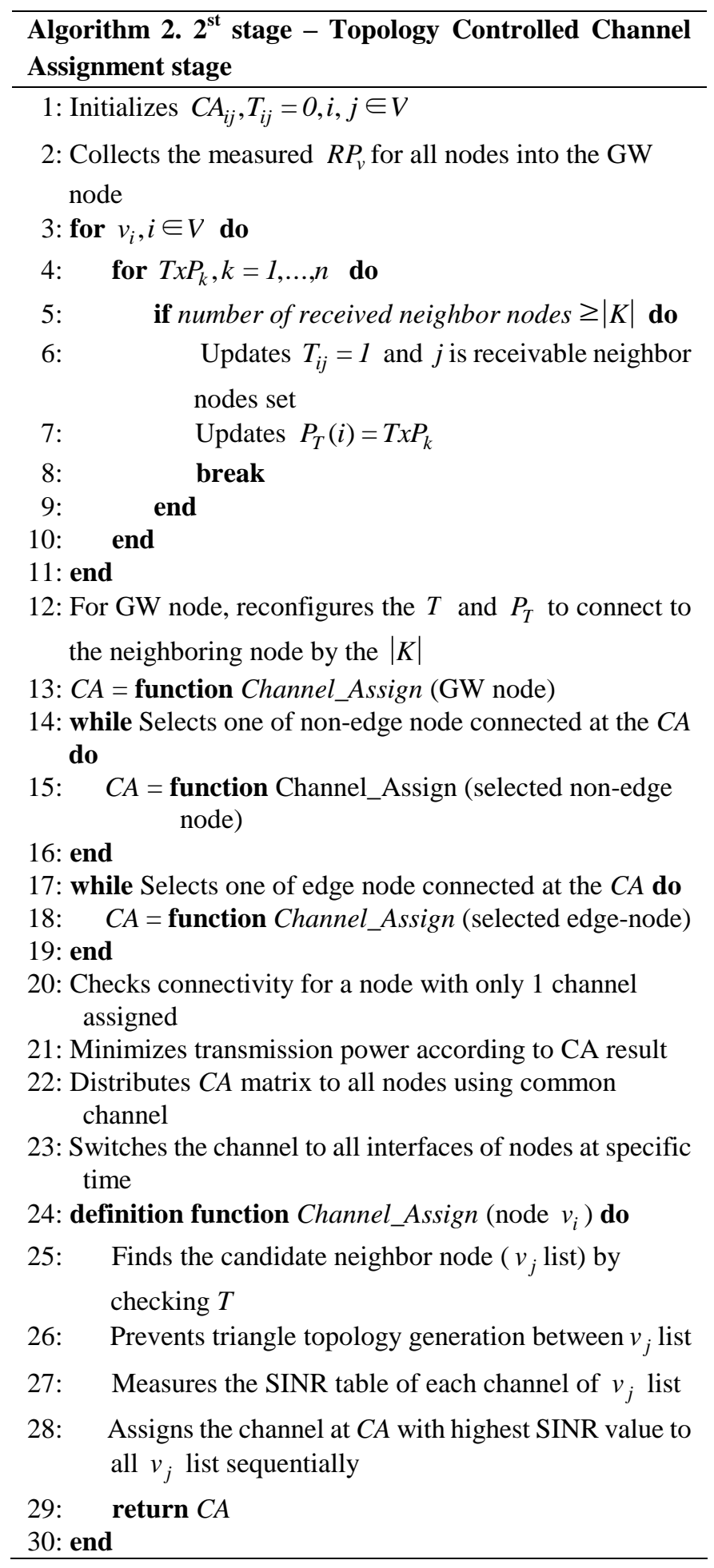

The 20th command line is to check connectivity for a node that only one channel is allocated. Fig. 2 shows the one step transmission power extension of node ' $\mathrm{B}$ ' to connect node ' $\mathrm{A}$ ' which has only one assigned channel. By increasing the transmission power slightly, it is possible not only to strengthen the connectivity of node ' $\mathrm{A}$ ' but also to distribute the traffic flows. Then, it minimizes transmission power of the node with unnecessarily high transmission power based on the $C A$ result. After doing above procedures, the GW node transmits the finally obtained $C A$ matrix to all nodes using the common channel. Finally, each node changes the assigned channel from the $C A$ matrix to the all interfaces of each node at a particular time.

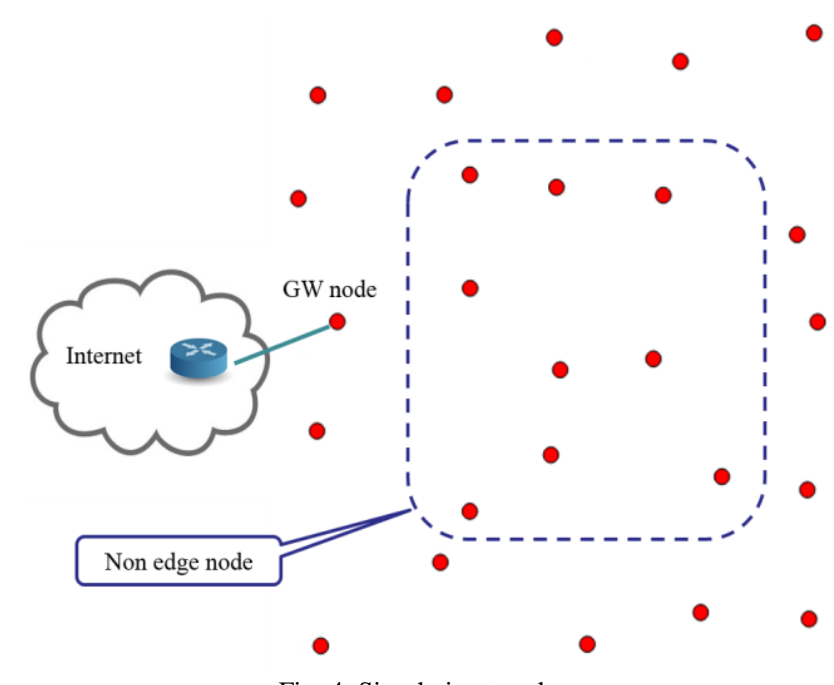

Fig. 4. Simulation topology.

The 24th to 30th command lines are the channel assignment function of the input node. First, because the transmission power of each node is not the same, we find a list of neighbor nodes to which channels must be allocated based on $T$. Then, an explanation for preventing triangle topology generation is shown in Fig. 3. As shown in (a) of Fig. 3, links 'A-B' and 'A-C' are originally connected. When the node ' $\mathrm{C}$ ' allocates a channel $I D c_{i}$ to the link 'B-C' as shown in (b) of Fig. 3, a triangle topology is created. By doing so, it not only causes more co-channel interference in a narrow area but also degrades network connectivity by using a link unnecessarily. For this reason, the node ' $C$ ' sets a channel with a high SINR value to one of the links ('A-C' or 'B-C') as shown in (c) of Fig. 3. After preventing the triangle topology generation, a channel with best SINR is allocated to each neighbor node.

\section{PERFORMANCE EVALUATION}

In this section, we evaluate our proposed TCCA algorithm using the NS-3 network simulator [6], [7]. We conducted performance analysis according to transmission power, traffic pattern, and routing metric (the proactive and the reactive mode). The performance metrics are throughput and average end-to-end delay according to traffic load.

Fig. 4 shows the 25 node WMN topology for our simulation which consists of 24 general mesh nodes and one GW mesh node located at the edge of the WMN. If a WMN operator configures the mesh routers of the WMN randomly, it may cause not only inefficient assignment of the channel but also have a significant performance difference depending on the network topology. Therefore, we have configured WMN mesh routers with the policies. First, all nodes are spaced every 170 meters in 5 by 5 grid topology. Since the real network cannot be placed in a grid topology due to a geographical problem, each node is repositioned with a uniformly random value between -60 and 60 meters at that location. Each node is classified into non-edge node and edge node as shown in Fig. 4. Since traffic is concentrated on non-edge nodes rather than edge nodes, we preferentially configure the non-edge nodes and allocate channels to non-edge nodes. 


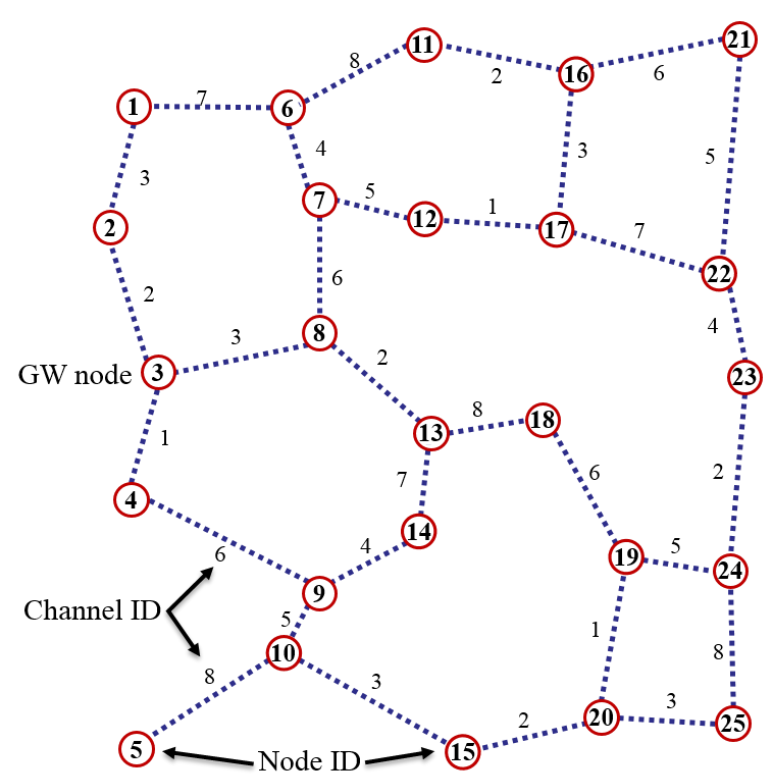

Fig. 5. Channel assignment result of TCCA algorithm in a given topology.

In the simulation, each node has three $802.11 \mathrm{a} 6 \mathrm{Mbps}$ interfaces, and eight non-overlapping orthogonal channels. We use the log-distance propagation with an exponential variable of 2.7 as a loss model. The threshold of energy detection value is $-89 \mathrm{dBm}$. The noise figure, which is defined as the difference between the noise output of the actual receiver to the noise output of an ideal receiver with the same gain and bandwidth, is $7 \mathrm{~dB}$.

The algorithm 1 is performed for the first 5 seconds, after that the algorithm 2 is executed for 10 seconds during simulation time. After that, we generate $100 \mathrm{TCP}$ traffic flows with exponentially distributed durations for 100 seconds of simulation time. There are two type of traffic pattern. The first is called 50:50 traffic that half of traffic is in/out traffic through GW node, the rest half is P2P traffic in WMN. The other is called 0:100 traffic that all of traffic are $\mathrm{P} 2 \mathrm{P}$ traffic in WMN. We evaluate the HWMP routing protocol reactive and proactive modes.

Both Fig. 5 and Table I show the topology controlled channel assignment result of TCCA algorithm. As shown in Fig. 5, channels are evenly assigned, because the TCCA algorithm allocates the channel with the highest SINR value. Also, as shown in Table I , the transmission power of each node is also taken into consideration of the distance to neighboring nodes and channel interference. For example, the transmission power of node 7 need not be greater than $14 \mathrm{dBm}$ to connect with neighboring nodes (node 6, 8, 12). However, in order for the node 21 to connect to the node 16 and 22, the transmission power must be at least $21 \mathrm{dBm}$.

TABLE I: TRANSMISSION POWER OF EACH NODE

\begin{tabular}{c||lllllllll}
\hline \hline Node ID & 1 & 2 & 3 & 4 & 5 & 6 & 7 & 8 & 9 \\
\hline Tx power $(\mathrm{dBm})$ & 16 & 16 & 17 & 20 & 18 & 16 & 14 & 17 & 20 \\
\hline \hline Node ID & 10 & 11 & 12 & 13 & 14 & 15 & 16 & 17 & 18 \\
\hline Tx power $(\mathrm{dBm})$ & 19 & 16 & 14 & 15 & 13 & 19 & 17 & 17 & 17 \\
\hline \hline Node ID & 19 & 20 & 21 & 22 & 23 & 24 & 25 & & \\
\hline Tx power $(\mathrm{dBm})$ & 17 & 17 & 21 & 21 & 19 & 19 & 16 & & \\
\hline \hline
\end{tabular}

The TCCA algorithm prevents the creation of triangular structures. For example in Fig. 5, the topology configuration is efficiently performed by avoiding the generation of the triangular structure of the node sets such as $(7,8,12),(9,10$, $14)$, and $(13,14,18)$. Also, the TCCA algorithm considers the connectivity of the node which has only one channel. In Fig. 5, originally node 4 and node 15 had only one neighbor node. By increasing the transmission power of node 9 and node 10 by one step, links 4-9 and 10-15 are generated to ensure high connectivity of the WMN.

All of following simulation results are obtained by averaging ten simulation results for accuracy. Fig. 6 shows the throughput and end-to-end delay performance of HWMP proactive routing protocol according to the node transmission power obtained by TCCA algorithm (Table I) and the same transmission power of all node (called same power case which uses the same channel assignment scheme except for the power control part of TCCA algorithm).

The proposed TCCA algorithm shows slightly higher throughput than the same power case. In the delay performance case, the TCCA algorithm shows much better performance than the same power case. For the same power case, the performance is degraded because the topology is not optimized and the channel interferences is high. On the other hand, the TCCA algorithm guarantees the connectivity of GW node and manages the WMN topology by adjusting the transmission power of each node. Therefore, the average end-to-end delay performance of the TCCA algorithm and the same power case shows a remarkable difference. Since the HWMP proactive mode is root-based routing, both performance is degraded when traffic is concentrated in the GW mesh node.
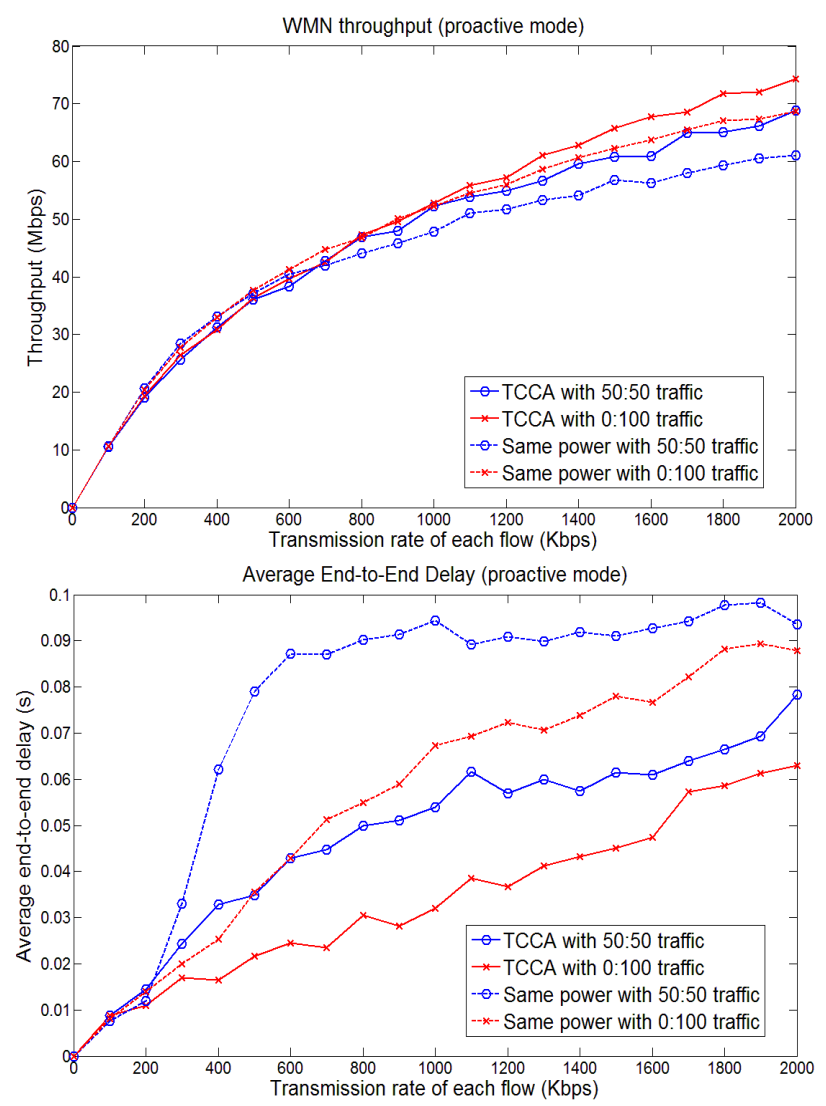

Fig. 6. Performance of the HWMP proactive routing mode. 

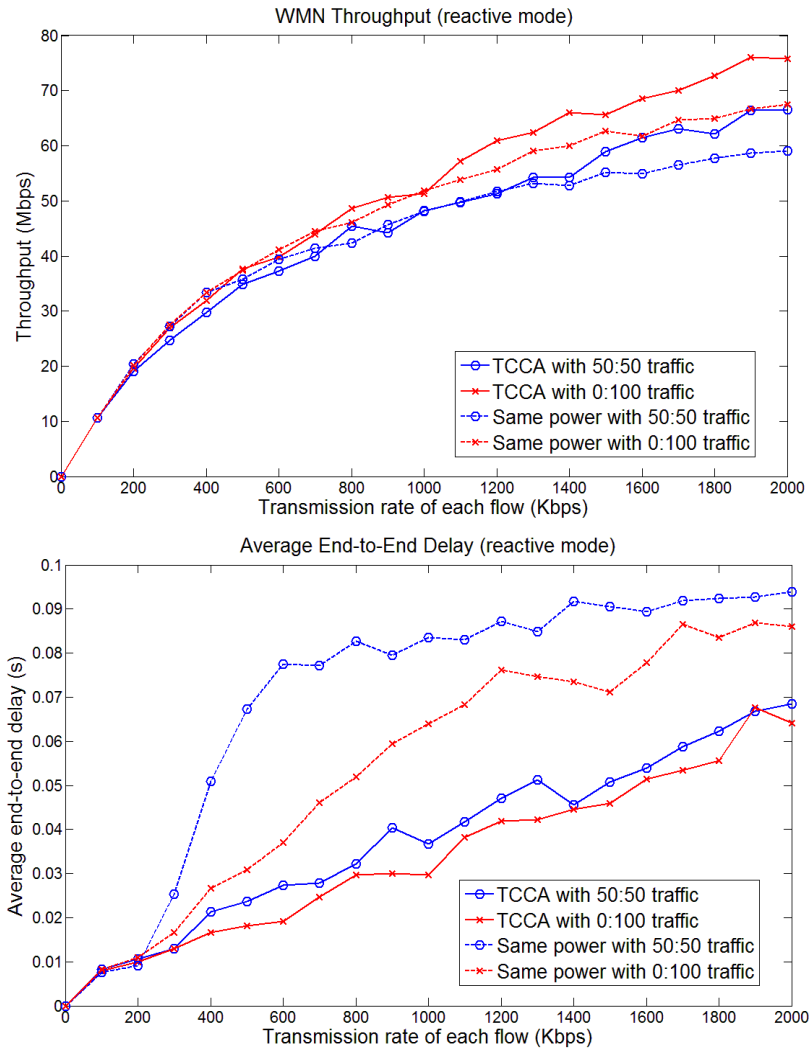

Fig. 7. Performance of the HWMP reactive routing mode.

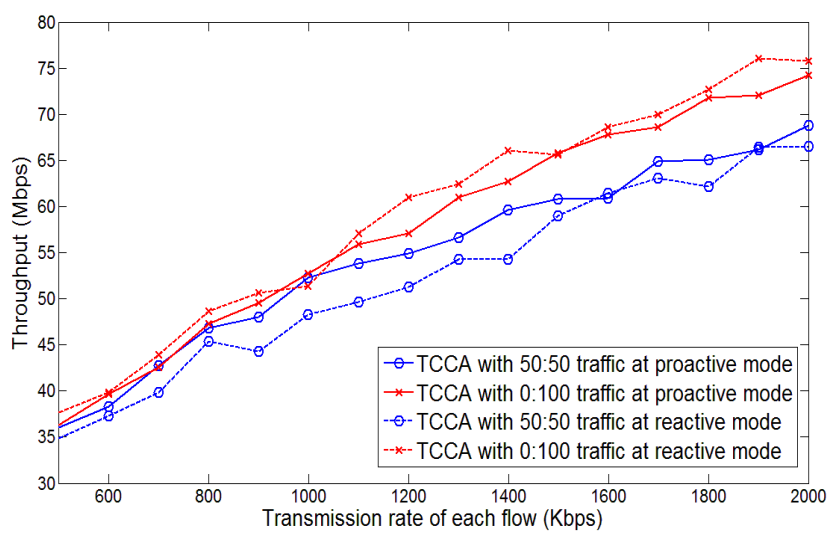

Fig. 8. Throughput comparison of the proactive and the reactive routing protocol.

Fig. 7 shows the throughput and end-to-end delay performance of the HWMP reactive routing protocol. Both performances in the reactive mode show the similar tendency to the proactive mode. The on-demand based reactive mode shows better performance when the most of traffic pattern is P2P traffic than the GW traffic. In addition, unlike the proactive mode, the reactive mode has low delay performance, because traffic is not concentrated in the GW neighbor nodes but spreads throughout the network.

Fig. 8 shows the throughput comparison of two routing protocols. For the 50:50 traffic pattern, the proactive mode shows higher throughput than the reactive mode. The reactive mode shows slightly better throughput performance than the proactive mode in case of 0:100 traffic pattern. Therefore, the TCCA algorithm can also be adapted to the traffic pattern change in real time, by selecting the proactive mode when traffic is concentrated on the gateway and by selecting the reactive mode when $\mathrm{P} 2 \mathrm{P}$ traffic is high.

\section{CONCLUSION}

In this paper, we proposed the topology controlled channel assignment algorithm with power control (TCCA) algorithm. The TCCA algorithm, which constructs the topology by controlling mesh node transmission power using measured received power and assigns the channel with low interference using SINR, can be applied to various industrial fields in real time basis. Simulation studies showed that the TCCA algorithm outperforms the same transmission power case by guaranteeing $\mathrm{GW}$ node connectivity and preventing unnecessary link generation. Also, the TCCA algorithm can support various traffic patterns by suitably selecting one of HWMP routing protocols. In the future work, we will develop energy efficiency TCCA algorithm that can consider the both energy consumption of the nodes and links.

\section{REFERENCES}

[1] I. Akyildiz and X. Wang, "A survey on wireless mesh networks," IEEE Communications Magazine, vol. 43, no. 9, 2005.

[2] A. Islam and A. Alim, "Channel assignment techniques for multi-radio wireless mesh networks: A survey," IEEE Communications Surveys \& Tutorials, vol. 18, no. 2, 2016.

[3] M. Mahesh, Samir, and A. Subramanian, "A topology control approach for utilizing multiple channels in multi-radio wireless mesh networks," Computer Networks, vol. 54, no. 2, pp. 241-256, 2010.

[4] C. Aizaz, N. Ahmad, and R. Hafez, "Improving throughput and fairness by improved channel assignment using topology control based on power control for multi-radio multi-channel wireless mesh networks," EURASIP Journal on Wireless Communications and Networking, pp. 1-25, 2012.

[5] 802.11-2012, "IEEE standard for information technology Telecommunications and information exchange between systems local and metropolitan area networks - Specific requirements," Wireless LAN Medium Access Control (MAC) and Physical Layer (PHY) Specifications.

[6] The NS-3 network simulator. [Online]. Available: http://www.nsnam.org

[7] A. Kirill and P. Boyko, "Ieee 802.11 s mesh networking ns-3 model," International Proceedings of ns3, 2010.

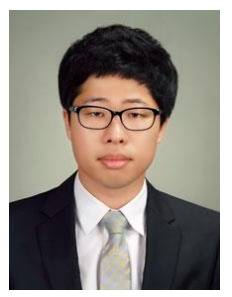

Sang-Hyun Lee was born in 1988 in South Korea. He received the B.S. and M.S. degrees from the Korea Advanced Institute of Science and Technology (KAIST), Daejeon, South Korea, in electrical engineering in 2011 and 2013. He is currently studying for a Ph.D. degree at KIAST. His research mainly focus on wireless mesh network, energy saving techniques, and network protocols.

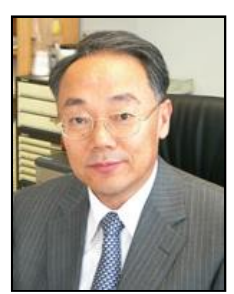

Hong-Shik Park was born in 1953 in South Korea. He received the B.S. degree from Seoul National University, Seoul, South Korea, in 1977, and the M.S. and $\mathrm{Ph} . \mathrm{D}$. degrees from the Korea Advanced Institute of Science and Technology (KAIST), Daejeon, South Korea, in electrical engineering in 1986 and 1995. In 1977, he joined the Electronics and Telecommunication Research Institute (ETRI) where he worked on the Development of the TDX Digital Switching System Family, including TDX-1, TDX-1A, TDX-1B, TDX-10, and ATM Switching Systems. In 1998, he moved to the Information and Communications University, Daejeon, South Korea, as a faculty member. Currently, he is a professor in the Department of Electrical and Electronics Engineering, KAIST, Daejeon, South Korea. His research interests are network architecture, network protocols, and the performance analysis of telecommunication systems. He is a member of the IEEE, IEEK, and KICS of South Korea. 